\title{
Primordial black holes and secondary gravitational waves from string inspired general no-scale supergravity
}

\author{
Lina $\mathrm{Wu},{ }^{1, *}$ Yungui Gong, ${ }^{2, \dagger}$ and Tianjun $\mathrm{Li}^{1,3,4, \ddagger}$ \\ ${ }^{1}$ School of Sciences, Xi'an Technological University, Xi'an 710021, China \\ ${ }^{2}$ School of Physics, Huazhong University of Science and Technology, Wuhan, Hubei 430074, China \\ ${ }^{3}$ CAS Key Laboratory of Theoretical Physics, Institute of Theoretical Physics, \\ Chinese Academy of Sciences, Beijing 100190, China \\ ${ }^{4}$ School of Physical Sciences, University of Chinese Academy of Sciences, \\ No. 19A Yuquan Road, Beijing 100049, China
}

The formation of primordial black hole $(\mathrm{PBH})$ dark matter and the generation of scalar induced secondary gravitational waves (SIGWs) have been studied in the generic no-scale supergravity inflationary models. By adding an exponential term to the Kähler potential, the inflaton experiences a period of ultraslow-roll and the amplitude of primordial power spectrum at small scales is enhanced to $\mathcal{O}\left(10^{-2}\right)$. The enhanced power spectra of primordial curvature perturbations can have both sharp and broad peaks. A wide mass range of PBHs can be produced in our model, and the frequencies of the accompanied SIGWs are ranged form nanohertz to kilohertz. We show four benchmark points where the generated PBH masses are around $\mathcal{O}\left(10^{-16} M_{\odot}\right), \mathcal{O}\left(10^{-12} M_{\odot}\right), \mathcal{O}\left(10^{-2} M_{\odot}\right)$ and $\mathcal{O}\left(10^{2} M_{\odot}\right)$. The PBHs with masses around $\mathcal{O}\left(10^{-16} M_{\odot}\right)$ and $\mathcal{O}\left(10^{-12} M_{\odot}\right)$ can make up almost all the dark matter, and the accompanied SIGWs can be probed by the upcoming space-based gravitational wave observatory. Also, the SIGWs accompanied with the formation of stellar mass PBHs can be used to interpret the stochastic GW background in the nanohertz band, detected by the North American Nanohertz Observatory for gravitational waves, and can be tested by future interferometric gravitational wave observatory.

\footnotetext{
*wulina@xatu.edu.cn

$\dagger$ yggong@hust.edu.cn

$\ddagger$ tli@itp.ac.cn
} 


\section{INTRODUCTION}

Since the direct detections of gravitational wave $(\mathrm{GW})$ by the Laser Interferometer Gravitational-Wave Observatory (LIGO) Scientific Collaboration and the Virgo Collaboration [1-13], the idea that primordial black hole $(\mathrm{PBH})$ can be considered as dark matter (DM) candidate [14-22] has again attracted the attention of physicists and astronomers [2332]. The DM fraction in the form of PBHs is tightly constrained by current observations $[28,33,34]$, but there is no observational constraint in the mass windows around the masses $10^{-12} M_{\odot}$ and $10^{-16} M_{\odot}$, so PBHs with masses around $10^{-12} M_{\odot}$ and $10^{-16} M_{\odot}$ can account for the total amount of DM.

PBHs are formed during radiation domination through gravitational collapse in overdense regions, where the density contrast of small-scale overdense regions at horizon reentry is greater than the threshold value $[35,36]$. To produce PBHs during the radiation era, the primordial scalar power spectrum at small scales should be enhanced to $\mathcal{O}(0.01)$ [37, 38]. However, the amplitude of the scalar power spectrum at $k_{*}=0.05 \mathrm{Mpc}^{-1}$ is constrained to be $\mathcal{P}_{\zeta}\left(k_{*}\right)=2.10 \times 10^{-9}[39,40]$. Thus, how to enlarge the primordial curvature perturbation at small scales becomes the key to progress. One mechanism to realize the enhancement of the power spectrum is the ultraslow-roll inflation with an inflection point [41-56]. The other mechanisms rely on a peak function either in the nonminimal coupling or in noncannonical kinetic term [57-67]. The location of the inflection point or the peak in the primordial scalar power spectrum is crucial for the calculation of the mass and abundance of PBHs. Near the inflection point, the inflaton potential $V(\phi)$ has an extreme flat plateau where the slow-roll parameter $\epsilon_{1}$ becomes very small and the power spectrum amplifies. However, the slow-roll approximation $\left|\epsilon_{2}\right| \ll 1$ is not satisfied around the point. To describe the precise evolution of the power spectrum, the Mukhanov-Sasaki equation should be numerically solved for each mode $k$ [68].

Since the scalar perturbations and tensor perturbations are coupled at the nonlinear level, the large primordial curvature perturbations at small scales will induce second-order tensor perturbations after the horizon reentry. The scalar induced secondary GWs (SIGWs) accompanied with the formation of PBH have been extensively studied [69-91]. The large curvature perturbations are the sources of both SIGWs and PBHs, and hence GW observations will place limit on the abundance of PBHs. The stochastic GW background detected by 
pulsar timing arrays (PTA) from North American Nanohertz Observatory (NANOGrav) [92] can be explained by SIGWs accompanied with the formation of solar mass PBHs [22, 93, 94]. The SIGWs accompanied by PBHs with masses around $\mathcal{O}\left(10^{-12} M_{\odot}\right)$ can be testable with the space-based GW observatory like Laser Interferometer Space Antenna (LISA) [95], Taiji [96], and TianQin [97].

Inflationary models and PBHs [98-105] have been studied before in the simple no-scale supergravity (one modulus model) [106, 107], which can be realized via the Calabi-Yau compactification with standard embedding of the weakly coupled heterotic $E_{8} \times E_{8}$ theory [108] and M-theory on $S^{1} / Z_{2}$ [109]. Moreover, one of us (TL) has studied various orbifold compactifications of M-theory on $T^{6} / Z_{3}, T^{6} / Z_{6}, T^{6} / Z_{12}$, as well as the compactification by keeping singlets under $S U(2) \times U(1)$ symmetry, and then the compactification on $S^{1} / Z_{2}$ [110], which provide the general frameworks for no-scale inflation. In this paper, with the general no-scale supergravity theories inspired by the above $T^{6} / Z_{12}$ orbifold compactification (three moduli model) and compactification by keeping singlets under $S U(2) \times U(1)$ symmetry (two moduli model) [110], we propose the generic inflationary models in which the PBH and SIGWs can be generated. We first discuss the simple and general no-scale supergravity theories, and show that the inflaton potential in three moduli models is similar to the global supersymmetry with canonical Kähler potential in Sec. II, but in fact they are completely different due to the Kähler potential differences. Then we study the corresponding inflationary models in Sec. III. In particular, we find that the tensor-to-scalar ratios in the two and three moduli models [110] are much smaller than that in the simple no-scale model or one modulus model $[108,109]$, and thus, the two and three moduli models might provide better frameworks to satisfy the swampland conjecture criteria [111, 112]. The detailed studies will be given elsewhere. In Sec. IV, we add an exponential term to the Kähler potential and show the enhancement of the scalar power spectrum by numerically solving the Mukhanov-Sasaki equation. Then we discuss the production of PBHs and SIGWs. The conclusions are drawn in Sec. V. In the following, we set the reduced Planck mass $M_{\mathrm{Pl}}^{2}=8 \pi G=1$.

\section{THE SIMPLE AND GENERAL NO-SCALE SUPERGRAVITY THEORIES}

The $\mathcal{N}=1$ supergravity Lagrangian can be written in the form

$$
\mathcal{L}=-\frac{1}{2} R+K_{i}^{\bar{j}} \partial_{\mu} \varphi^{i} \partial^{\mu} \bar{\varphi}_{\bar{j}}-V,
$$


where the Kähler metric is $K_{i}^{\bar{j}} \equiv \partial^{2} K /\left(\partial \varphi^{i} \partial \bar{\varphi}_{\bar{j}}\right)$. The effective scalar potential is

$$
V=e^{G}\left[\frac{\partial G}{\partial \varphi^{i}}\left(K^{-1}\right)_{\bar{j}}^{i} \frac{\partial G}{\partial \bar{\varphi}_{\bar{j}}}-3\right],
$$

where the Kähler function is $G \equiv K+\ln |W|^{2}$, and $\left(K^{-1}\right) \frac{i}{j}$ is the inverse of the Kähler metric. Introducing the Kähler covariant derivative

$$
D_{i} W \equiv W_{i}+K_{i} W
$$

the scalar potential can be rewritten as

$$
V=e^{K}\left[D_{i} W\left(K^{-1}\right)_{\bar{j}}^{i} D^{\bar{j}} \bar{W}-3|W|^{2}\right]
$$

\section{A. The simple no-scale supergravity: One modulus model}

The Kähler potential is

$$
K=-3 \ln \left(T_{1}+\bar{T}_{1}-|\varphi|^{2}\right)
$$

The Kähler metric and the inverse of the Kähler metric are

$$
\begin{gathered}
K_{i}^{\bar{j}}=\frac{3}{X^{2}}\left(\begin{array}{cc}
1 & -\varphi \\
-\bar{\varphi} & X_{1}
\end{array}\right), \\
\left(K^{-1}\right)_{\bar{j}}^{i}=\frac{X}{3}\left(\begin{array}{cc}
X_{1} & \bar{\varphi} \\
\varphi & 1
\end{array}\right),
\end{gathered}
$$

with $X=T_{1}+\bar{T}_{1}-|\varphi|^{2}$ and $X_{i}=T_{i}+\bar{T}_{i}(i=1,2,3)$.

We consider the superpotential with a single chiral superfield as

$$
W=\frac{M}{2} \varphi^{2}-\frac{\lambda}{3} \varphi^{3}
$$

which reduces to the Wess-Zumino model with the potential $V=A \phi^{2}(v-\phi)^{2}$ when the imaginary part of the scalar component of $\varphi$ vanishes [100]. In this case, $W_{T_{1}}=0^{1}$ and

$$
D_{i} W=W\left(-\frac{3}{X} \frac{3}{X} \bar{\varphi}+\frac{W_{\varphi}}{W}\right) .
$$

Then, the scalar potential becomes

$$
V=\frac{\left|W_{\varphi}\right|^{2}}{3 X^{2}}
$$

\footnotetext{
1 The $\eta$-problem is avoided since no large mass term is generated $[113,114]$.
} 


\section{B. The $S U(2) \times U(1)$ symmetry: Two moduli model}

We consider the compactification of M-theory by keeping singlets under $S U(2) \times U(1)$ symmetry, and then the compactification on $S^{1} / Z_{2}$ [110], which has two moduli. The Kähler potential is

$$
K=-2 \log \left(T_{1}+\bar{T}_{1}-|\varphi|^{2}\right)-\log \left(T_{2}+\bar{T}_{2}\right),
$$

where for simplicity we neglect the irrelevant scalar fields. The Kähler metric is

$$
K_{i}^{\bar{j}}=\frac{2}{X^{2} X_{2}^{2}}\left(\begin{array}{ccc}
X_{2}^{2} & 0 & -X_{2}^{2} \varphi \\
0 & X^{2} / 2 & 0 \\
-X_{2}^{2} \bar{\varphi} & 0 & X_{1} X_{2}^{2}
\end{array}\right) .
$$

Using the superpotential (7), the covariant derivative becomes

$$
D_{i} W=W\left(-\frac{2}{X}-\frac{1}{X_{2}} \frac{2}{X} \bar{\varphi}+\frac{W_{\varphi}}{W}\right)
$$

with $W_{T_{i}}=0$. Then the scalar potential becomes

$$
V=\frac{\left|W_{\varphi}\right|^{2}}{2 X X_{2}}
$$

\section{The $T^{6} / Z_{12}$ orbifold compactification: Three moduli model}

In this subsection, we consider the $T^{6} / Z_{12}$ orbifold compactification of M-theory, and then the compactification on $S^{1} / Z_{2}$ [110], which has three moduli. The Kähler potential is

$$
K=-\log \left(T_{1}+\bar{T}_{1}-|\varphi|^{2}\right)-\sum_{m=2,3} \log \left(T_{m}+\bar{T}_{m}\right),
$$

where for simplicity we neglect the irrelevant scalar fields as well. The Kähler metric is

$$
K_{i}^{\bar{j}}=\frac{1}{X^{2} X_{2}^{2} X_{3}^{2}}\left(\begin{array}{cccc}
X_{2}^{2} X_{3}^{2} & 0 & 0 & -X_{2}^{2} X_{3}^{2} \varphi \\
0 & X^{2} X_{3}^{2} & 0 & 0 \\
0 & 0 & X^{2} X_{2}^{2} & 0 \\
-X_{2}^{2} X_{3}^{2} \bar{\varphi} & 0 & 0 & X_{1} X_{2}^{2} X_{3}^{2}
\end{array}\right) .
$$

Using the superpotential (7), the covariant derivative becomes

$$
D_{i} W=W\left(-\frac{1}{X}-\frac{1}{X_{2}}-\frac{1}{X_{3}} \frac{1}{X} \bar{\varphi}+\frac{W_{\varphi}}{W}\right),
$$


with $W_{T_{i}}=0$. Then the scalar potential becomes

$$
V=\frac{\left|W_{\varphi}\right|^{2}}{X_{2} X_{3}}
$$

Thus, after we fix $T_{2}$ and $T_{3}$, we obtain the inflationary model similar to that with the global supersymmetry.

\section{General parametrization}

To study inflation, PBHs, and SIGWs, we parametrize the generic Kähler potential as follows

$$
\begin{aligned}
K= & -N_{X} \log \left(T_{1}+\bar{T}_{1}-|\varphi|^{2}\right)-N_{Y} \log \left(T_{2}+\bar{T}_{2}\right) \\
& -N_{Z} \log \left(T_{3}+\bar{T}_{3}\right),
\end{aligned}
$$

where $N_{X}+N_{Y}+N_{Z}=3$. In particular, the discussions for inflation, PBHs and SIGWs for the $T^{6} / Z_{12}$ orbifold compactification are similar to the scenario with $N_{X}=2, N_{Y}=1$, and $N_{Z}=0$. Using the superpotential (7), the covariant derivative becomes

$$
D_{i} W=W\left(-\frac{N_{X}}{X}-\frac{N_{Y}}{X_{2}}-\frac{N_{Z}}{X_{3}} \frac{N_{X} \bar{\varphi}}{X}+\frac{W_{\varphi}}{W}\right) .
$$

Thus, the general scalar potential can be written as

$$
V=\frac{\left|W_{\varphi}\right|^{2}}{N_{X} X^{N_{X}-1} X_{2}^{N_{Y}} X_{3}^{N_{Z}}}
$$

\section{THE INFLATIONARY MODELS}

We assume that all the real components of the complex fields which do not drive inflation have been stabilized, whereas the inflaton field remains dynamical. Here we fix the modulus $T_{i}$ with the vacuum expectation value $(\mathrm{VEV}) 2\left\langle\operatorname{Re}\left(T_{i}\right)\right\rangle=c_{i}$ and $\left\langle\operatorname{Im}\left(T_{i}\right)\right\rangle=0$ [104], and choose the inflationary trajectory along with $\bar{\varphi}=\varphi$. For the simple no-scale supergravity, the scalar potential in Eq. (9) becomes

$$
V_{1}=\frac{M^{2} \varphi^{2}(1-d \varphi)^{2}}{3\left(c_{1}-\varphi^{2}\right)^{2}},
$$

where $d=\lambda / M$. One notes that the kinetic term in Eq. (1) is noncanonical, so we need to define a new canonical field $\chi$, which satisfies

$$
\frac{1}{2} \partial_{\mu} \chi \partial^{\mu} \chi=K_{\varphi \varphi} \partial_{\mu} \varphi \partial^{\mu} \varphi
$$


By integrating the above equation, we get the field transformation $\varphi=\sqrt{c_{1}} \tanh (\chi / \sqrt{6})$. The Starobinsky model is realized by choosing the specific parameter $\lambda=\sqrt{3} M$ and $c_{1}=$ $1 / \sqrt{3}$ with the potential

$$
V_{1}=\frac{M^{2}}{4}\left(1-e^{-\sqrt{\frac{2}{3}} \chi}\right)^{2} .
$$

Similarly, the scalar potentials in the two and three moduli models are

$$
\begin{aligned}
& V_{2}=\frac{M^{2} \varphi^{2}(1-d \varphi)^{2}}{2 c_{2}\left(c_{1}-\varphi^{2}\right)}, \\
& V_{3}=\frac{M^{2} \varphi^{2}(1-d \varphi)^{2}}{c_{2} c_{3}} .
\end{aligned}
$$

Using the field transformation $\varphi=\sqrt{c_{1}} \tanh \left(\chi / \sqrt{2 N_{X}}\right)$, they can be rewritten as

$$
\begin{aligned}
& V_{2}=\frac{M^{2} e^{-\chi}\left(1-e^{\chi}\right)^{2}\left(1+e^{\chi}+\sqrt{c_{1}} d\left(1-e^{\chi}\right)\right)^{2}}{8 c_{2}\left(1+e^{\chi}\right)^{2}}, \\
& V_{3}=\frac{c_{1} M^{2}\left(1-e^{\sqrt{2} \chi}\right)^{2}\left(1+e^{\sqrt{2} \chi}+\sqrt{c_{1}} d\left(1-e^{\sqrt{2} \chi}\right)\right)^{2}}{c_{2} c_{3}\left(1+e^{\sqrt{2}} \chi\right)^{4}} .
\end{aligned}
$$

The Hubble slow-roll parameters are defined as

$$
\epsilon_{1}=-\frac{\dot{H}}{H^{2}}, \epsilon_{i+1}=\frac{\dot{\epsilon}_{i}}{H \epsilon_{i}}, i=1,2,3,
$$

where a dot denotes the derivative with respect to time $t$. The spectral index $n_{s}$ and the tensor-to-scalar ratio $r$ in terms of slow-roll parameters are

$$
n_{s}=1+2 \epsilon_{2}-4 \epsilon_{1}, \quad r=16 \epsilon_{1} .
$$

Under the slow-roll condition, we calculate the observables $n_{s}$ and $r$ with $N=[50,60]$ and the results are shown in Fig. 1. The tensor-to-scalar ratio $r$ in these models is much smaller than the Planck 2018 constraint $r_{0.002}<0.056$ (95\% CL) [39] and the BICEP/Keck constraint $r_{0.05}<0.036$ (95\% CL) [115]. Thus, the tensor-to-scalar ratio in the models added by an exponential term in the Kähler potential will be tested by the coming GW observatories. In particular, we find that the tensor-to-scalar ratios in the two and three moduli models [110] are much smaller than that in the simple no-scale model or one modulus model [108, 109], so the two and three moduli models might provide better frameworks to satisfy the swampland conjecture criteria [111, 112]. The detailed study will be given elsewhere.

In the next section, we will discuss the enhancement of the primordial power spectrum at small scales by introducing an inflection point in the inflaton potential. Around the inflection 


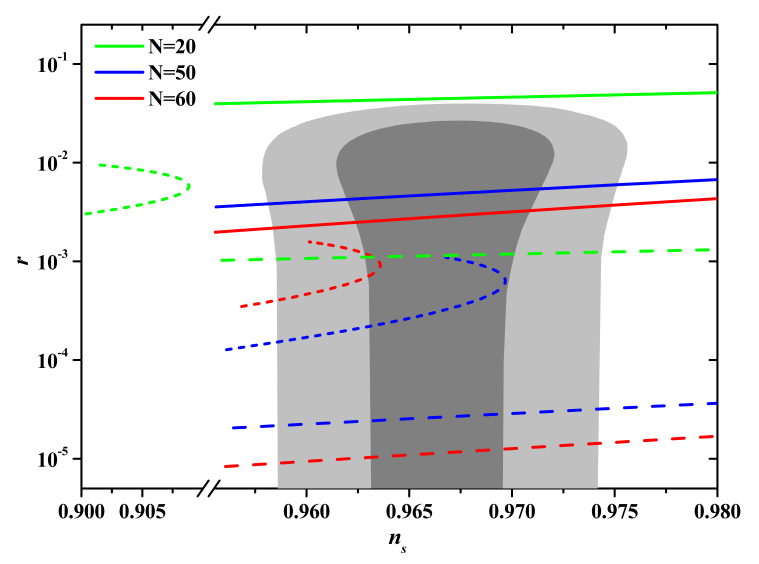

FIG. 1. Observables $n_{s}$ and $r$ along with constraints from Planck 2018 [39, 115]. The solid, dashed, and dotted line corresponds to the model with one Eq. (23), two Eq. (26), and three Eq. (27) moduli, respectively.

point, the potential has an extremely flat plateau, the slow-roll parameter $\epsilon_{1}$ becomes very small, and the slow-roll parameter $\left|\epsilon_{2}\right|$ becomes large, so the friction force becomes a driving force and the primordial power spectrum is enhanced, at the same time the number of $e$-folds also increases dramatically. To solve the problems of standard big bang cosmology, inflation has to last $50-60 e$-folds. For the convenience of discussion, we divide the total number of $e$-folds into two parts: the slow-roll regime $\delta N$ and the ultraslow-roll regime $\Delta N$. The width of the enhanced power spectrum is related to the value of $\Delta N$ or $\delta N$. If we want to get an enhanced power spectrum with a broad power spectrum, the ultraslow-roll regime $\Delta N$ should be bigger and the slow-roll regime $\delta N$ should be less than $30 e$-folds. Less slowroll inflation will make it harder for the model satisfying the constraints from the cosmic microwave background (CMB) observations. In Fig. 1, we show the results for $n_{s}$ and $r$ with $\delta N=20$. As seen from Fig. 1, either the scalar spectral $n_{s}$ or the tensor-to-scalar ratio $r$ for the models with one and three moduli model is inconsistent with the observational constraints if $\delta N=20$. Since the ultraslow-roll $\Delta N$ could have a wide range in the model with two moduli which makes the model easily to realize an enhanced power spectrum without violating the CMB constraints, thus we discuss PBHs and SIGWs produced in the model with two moduli only. 


\section{PBH AND SIGWS FROM THE MODIFIED KÄHLER POTENTIAL}

In this section, we show the enhancement of primordial scalar power spectrum at small scales, by adding an exponential term to the Kähler potential. In this way, an inflection point will be brought into the scalar potential. Inflaton will go through a period of ultraslow-roll inflation and the amplitude of the primordial power spectrum is enhanced to $\mathcal{O}\left(10^{-2}\right)$. The Kähler potential in Eq. (18) is modified as

$$
\begin{aligned}
K= & -2 \log \left[T_{1}+\bar{T}_{1}-|\varphi|^{2}+a e^{-b\left(\varphi^{\alpha}+\bar{\varphi}^{\alpha}\right)}\left(\varphi^{\beta}+\bar{\varphi}^{\beta}\right)\right] \\
& -\log \left[T_{2}+\bar{T}_{2}\right],
\end{aligned}
$$

where $a, b$ and $\alpha, \beta$ are real numbers. For simplification, we set $\alpha=2, \beta=2$. The parameters $(\alpha, \beta)$ can also take other integer numbers, like $(1,2)$, and $(2,2)$. The corresponding equations are similar to the model with $\alpha=2, \beta=2$, so we do not display them. The added exponential term differs from that in Refs. $[102,105]$ since the parameter $a$ is a negative real number of $\mathcal{O}(0.1)$ and $b$ is a positive real number of $\mathcal{O}(10)$. The correction will not change the whole theory except introducing an inflection point in the effective potential.

The inflationary direction is set as $\bar{\varphi}=\varphi$, and $\bar{T}_{i}=T_{i}=c_{i} / 2$. In order to study the scalar effective potential, we need to define a new canonical scalar field $\chi$ with the transformation $\mathrm{d} \chi / \mathrm{d} \varphi=\sqrt{2 K_{\varphi \varphi}}$. Therefore, the scalar potential of the inflaton $\varphi$ becomes

$$
V=V_{0} \frac{\varphi^{2} e^{4 b \varphi^{2}}(d \varphi-1)^{2}}{\left(e^{2 b \varphi^{2}}-8 a b \varphi^{2}\left(b \varphi^{2}-1\right)\right)\left(2 a \varphi^{2}+e^{2 b \varphi^{2}}\left(c-\varphi^{2}\right)\right)},
$$

with $c=c_{1}, V_{0}=M^{2} /\left(2 c_{2}\right)$ and $d=\lambda / M$. By integrating Eq. (22), we can get a generalized relation $\varphi(\chi)$ and the potential $V(\chi)$ numerically as shown in Fig. 2. One notes that an inflection point exists in the scalar potential with the addition of the extra exponential term in the Kähler potential. When the pivotal scale $k_{*}=0.05 \mathrm{Mpc}^{-1}$ leaves the horizon, $\chi_{*} \sim 2.5 M_{\mathrm{Pl}}$ or $\varphi_{*} \sim 0.32 M_{\mathrm{Pl}}$, so $e^{-b \varphi^{2}}$ is small if $b \sim 40$ and the effect of the exponential terms can be neglected. In this limit, the potential (31) reduces to the unmodified potential (24). In other word, the contribution from the extra exponential term can be ignored in the slow-roll regime.

We show four benchmark points in Table I, where the $e$-folding numbers are restricted to be $N \sim 50-60$. The parameters $c$ and $d$ affect the total $e$-folding number. It is interesting to note that the $e$-folding number spent around the inflection point is about $\Delta N \sim 15-40$, 
this is the reason why we can keep the remaining $e$-folding number before the end of inflation to be $N \sim 50-60$. For the model I, we plot the potential for the inflaton field $\chi$ in Fig. 2 . There is an inflection point at $\chi_{\mathrm{inf}}=0.8032 M_{\mathrm{Pl}}$, where the slow-roll conditions are no longer satisfied. The evolutions of the inflaton $\chi$ and the slow-roll parameters $\epsilon_{1}$ and $\epsilon_{2}$ in terms of the number of $e$-folds $N$ are shown in Fig. 3. The inflation ends at $\epsilon_{1}=1$ where the value of inflaton is $\chi_{e}=0.3936 M_{\mathrm{Pl}}$ for the model I. Near the inflection point at $\chi_{\mathrm{inf}}=0.8032 M_{\mathrm{Pl}}$, there is a plateau in the evolution of inflaton as a function of the number of $e$-folds $N$, the slow-roll parameters are $\epsilon_{1} \sim 10^{-7}$ and $\epsilon_{2} \sim 3$, and the inflation lasts almost $15 e$-folds. This is important for the enhancement of the scalar power spectrum, since the power spectrum under the slow-roll approximation is given by

$$
\mathcal{P}_{\zeta}(k) \simeq \frac{1}{12 \pi^{2}} \frac{V(\chi)}{\epsilon_{1}}
$$

By varying the parameter $b$, we can get an enhanced $\mathcal{P}_{\zeta}$ and then proper PBH abundance. However, this approximation in Eq. (32) fails to give us the accurate power spectrum near the inflection point. Thus, it is necessary to solve the Mukahanov-Sasaki equation numerically to obtain the accurate power spectrum.

\begin{tabular}{cccccccccc}
\hline \hline Model & $a$ & $b$ & $d / A$ & $\chi_{*}\left(M_{\mathrm{Pl}}\right)$ & $\chi_{p}\left(M_{\mathrm{Pl}}\right)$ & $\chi_{e}\left(M_{\mathrm{Pl}}\right)$ & $n_{s}$ & $r$ & $N$ \\
\hline I & -0.480 & 46.79179 & 1.0032 & 2.463 & 0.7781 & 0.3936 & 0.9603 & $1.1 \times 10^{-4}$ & 50.9 \\
II & -0.480 & 46.7649 & 1.0031 & 2.466 & 0.7782 & 0.3935 & 0.9652 & $1.8 \times 10^{-4}$ & 47.5 \\
III & -0.470 & 43.62443 & 1.0020 & 2.485 & 0.8272 & 0.4043 & 0.9627 & $2.1 \times 10^{-3}$ & 48.9 \\
IV & -0.467 & 42.3915 & 1.0 & 2.50 & 0.8481 & 0.4087 & 0.9772 & $6.9 \times 10^{-3}$ & 57.6 \\
\hline \hline
\end{tabular}

TABLE I. The model parameters with $c=0.14$ and the predicted observables $n_{s}$ and $r . \chi_{*}$ and $\chi_{e}$ correspond to the value of the inflaton at the horizon exit and the end of inflation. $\chi_{p}$ corresponds to value of the inflaton at which the power spectrum reaches the peak value.

The numerical results for $n_{s}$ and $r$ at the pivotal scale $k_{*}=0.05 \mathrm{Mpc}^{-1}$ are shown in Table I, and they are consistent with the CMB constraints $n_{s}=0.9649 \pm 0.0042(68 \%$ CL) and $r_{0.05}<0.036(95 \% \mathrm{CL})[39,115]$. The mass and abundance of PBHs are sensitive to the parameters $a$ and $b$. For a fixed $c$, a smaller $|a|$ will give a more broad peak for $\mathcal{P}_{\zeta}$ and then produce PBHs with a larger peak mass. When $d$ is slightly bigger than $A=\sqrt{27 / 32 c}$, the $e$-folding number increases rapidly and $r$ decreases to $10^{-4}$. The parameter $V_{0}$ is fixed by the 


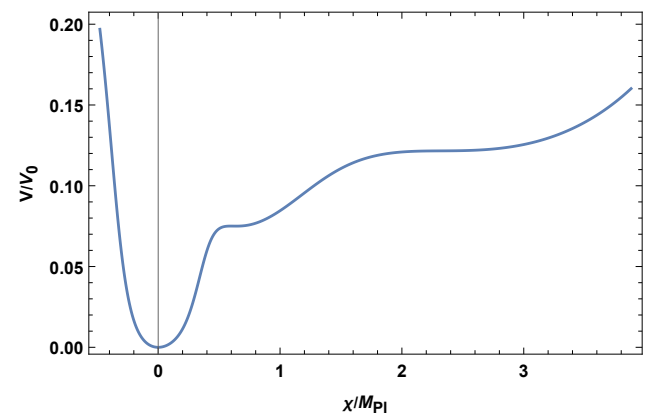

(a)

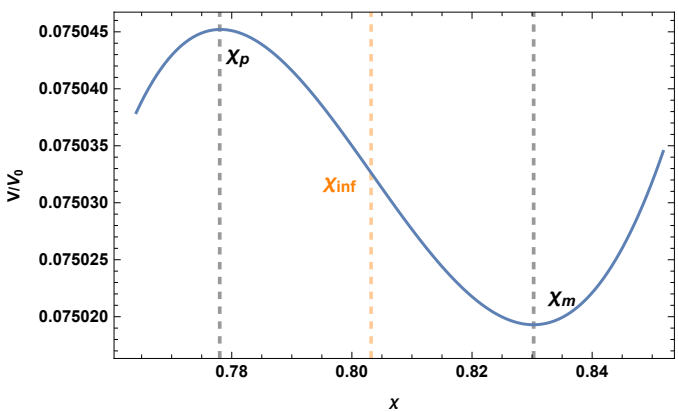

(b)

FIG. 2. (a) The potential $V(\chi)$ of the canonical scalar field $\chi$ for the model I with the model parameters as shown in Table I. (b) The upward step near the inflection point $\chi_{\mathrm{inf}}=0.8032 M_{\mathrm{Pl}}$. The power spectrum enhances from $\chi_{m}=0.8303 M_{\mathrm{Pl}}$ and peaks at $\chi_{p}=0.7781 M_{\mathrm{Pl}}$.

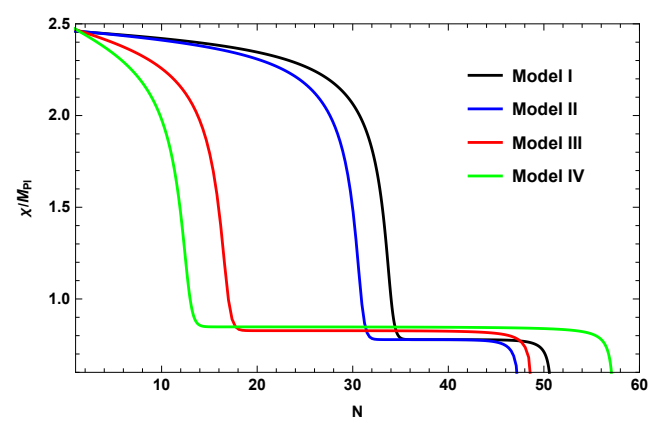

(a)

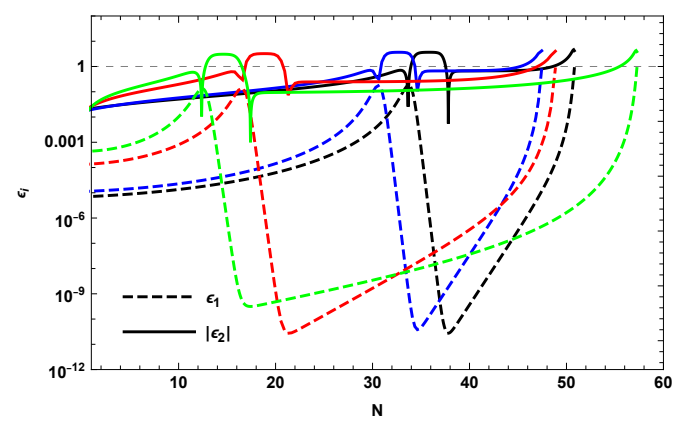

(b)

FIG. 3. The evolution of the inflaton $\chi$ (a) and the Hubble slow-roll parameters $\epsilon_{1}$ and $\left|\epsilon_{2}\right|$ (b) for the models shown in Table I. The inflation ends at $\epsilon_{1}(\chi)=1$.

amplitude of the power spectrum at the horizon crossing. The constraint on the amplitude of the power spectrum from Planck data is $\mathcal{P}_{\zeta}\left(k_{*}\right)=2.10 \times 10^{-9}[39,40]$.

From Fig. 3, we see that the number of $e$-folds near the inflection is about $\Delta N \simeq 15$ for the model I and II, whereas the number of $e$-folds is about $\Delta N \simeq 31$ for the model III and $\Delta N \simeq 40$ for the model IV. When the inflaton climbs an upward step in the potential from its minimum $\chi_{m}$ to the maximum $\chi_{p}$, the slow-roll parameter $\epsilon_{1}$ decreases and the power spectrum is enhanced. The climbing step is shown in the right panel of Fig. 2. Near the inflection point, the inflaton first decelerates and then accelerates when it rolls over the maximum point $\chi_{p}$ of the potential that gives the peak of the power spectrum. After the upward climbing, inflaton goes into the acceleration phase which decreases the power 
spectrum by the ratio $1 / \epsilon_{1}[116]$. As seen from Fig. 3 , if $\epsilon_{1}$ increases more slowly after $\chi_{p}$, then the inflaton stays near the inflection longer, and we get broader power spectrum. On the other hand, as shown in the right panel of Fig. 3 , the minimum value of $\epsilon_{1}$ in the model IV is bigger, so its peak value of the power spectrum is expected to be smaller.

\section{A. Primordial curvature perturbations}

Near the inflection point, the inflaton experiences a period of ultraslow-roll inflation and the amplitude of the primordial scalar power spectrum is enhanced. In order to obtain the power spectrum

$$
\mathcal{P}_{\zeta}=\frac{k^{3}}{2 \pi^{2}}\left|\frac{u_{k}}{z}\right|^{2}
$$

for the primordial curvature perturbation $\mathcal{R}$, we numerically solve the Mukhanov-Sasaki equation $[117,118]$

$$
u_{k}^{\prime \prime}+\left(k^{2}-\frac{z^{\prime \prime}}{z}\right) u_{k}=0
$$

for the scalar mode $u_{k}=-z \mathcal{R}$, where the prime means derivative with respect to the conformal time $\tau, z=\chi^{\prime} / H$ and $z^{\prime \prime} / z$ can be expressed in terms of the Hubble slow-roll parameters as

$$
\frac{z^{\prime \prime}}{z}=\mathcal{H}^{2}\left[2-\epsilon_{1}+\frac{3}{2} \epsilon_{2}-\frac{1}{2} \epsilon_{1} \epsilon_{2}+\frac{1}{4} \epsilon_{2}^{2}+\frac{1}{2} \epsilon_{2} \epsilon_{3}\right],
$$

with $\mathcal{H}=a H$. In the limit $k \rightarrow \infty$, the mode function $u_{k}$ takes the solution

$$
u_{k} \rightarrow \frac{1}{\sqrt{2 k}} e^{-i k \tau}
$$

We plot the numerical results for the power spectrum in Fig. 4 and show the peak values of the power spectrum in Table II. The black, red, blue, and green dashed lines correspond to the model I, II, III, and IV, respectively. Near the peak point $\chi_{p}$, the power spectrum is enhanced to $\mathcal{O}(0.01)$. The power spectrum in the models I and II has a sharp peak whereas the power spectrum in the models III and IV has a broad peak. The peak point $\chi_{p}$ in the model III and model IV are further away from the endpoint $\chi_{e}$ of inflaton than the other two models and the plateau in the potential of the inflaton $\chi$ in the models III and IV are more broader than the other two models. 


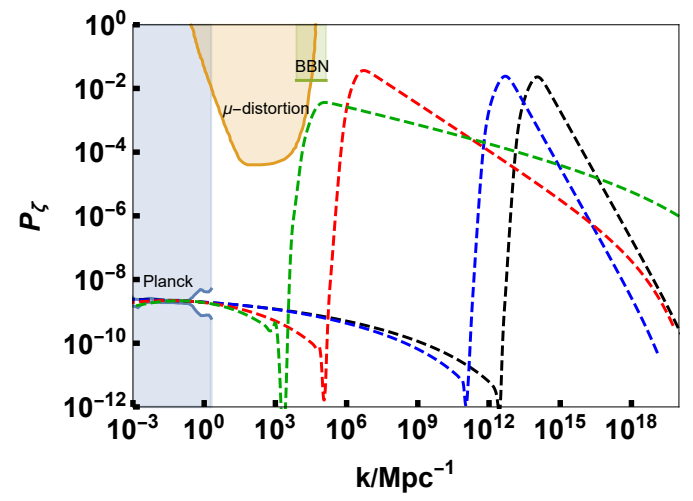

(a)

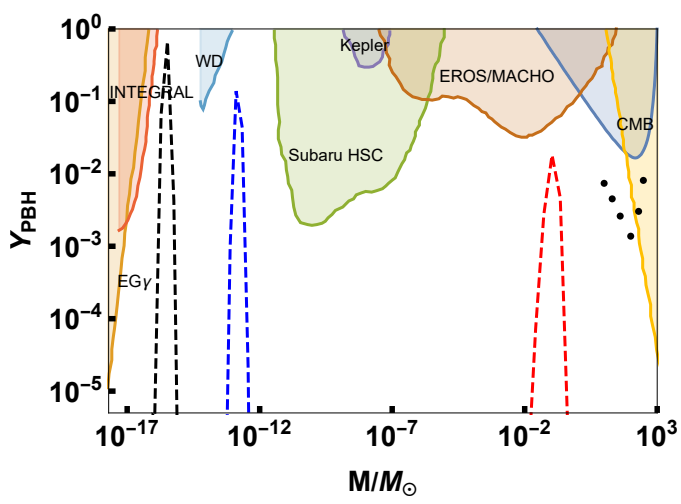

(b)

FIG. 4. The scalar power spectrum and PBH abundances. The black, blue, red, and green dashed lines correspond to the models I, II, III, IV, respectively. (a) The shaded constraints are from CMB observations [39], $\mu$-distortion of CMB [119], and the effect on the ratio between neutron and proton during the big bang nucleosynthesis (BBN) [79], respectively. (b) The shaded constraints are from the extragalactic gamma rays from $\mathrm{PBH}$ evaporation $(\mathrm{EG} \gamma)$ [120], galactic center 511 keV gamma-ray line (INTEGRAL), white dwarf explosion (WD) [121], microlensing events with Subaru HSC [86, 122], the Kepler satellite [123], EROS/MACHO [124], stochastic gravitational wave background by LIGO [125], LIGO merger rate [126], and accretion constraints from CMB $[127,128]$. The PBH abundance of Model IV is not shown in the figure since the peak abundance is only $10^{-83}$. It was argued that the constraint from WD explosion is not robust and the constraint can be removed [129].

\section{B. PBH formation}

The enhanced primordial curvature perturbations cause gravitational collapse in the overdense region at the horizon reentry during the radiation dominated era [28, 33, 62]. If the density fluctuation is larger than a centain threshold $\delta_{c}$, the gravity can overcome the pressure and hence $\mathrm{PBH}$ forms. The critical threshold $\delta_{c}$ for $\mathrm{PBH}$ formation has a wide range, from 0.07 to 0.7 [130-135]. In this paper, we take $\delta_{c}=0.4$ [136-139]. Assuming the primordial perturbations obey Gaussian statistics, the fractional energy density of PBHs at their formation time is given by the Press-Schechter formalism [140]

$$
\beta(M) \equiv \frac{\rho_{\mathrm{PBH}}}{\rho_{\mathrm{tot}}} \simeq \sqrt{\frac{2}{\pi}} \frac{\sqrt{P_{\zeta}}}{\mu_{c}} \exp \left(-\frac{\mu_{c}^{2}}{2 P_{\zeta}}\right),
$$




\begin{tabular}{cccccc}
\hline \hline Model & $k_{\text {peak }}\left(\mathrm{Mpc}^{-1}\right)$ & $\mathcal{P}_{\zeta}$ & $M_{\mathrm{PBH}}\left(M_{\odot}\right)$ & $Y_{\mathrm{PBH}}$ & $f_{\mathrm{GW}}(\mathrm{Hz})$ \\
\hline I & $1.05 \times 10^{14}$ & 0.023 & $3.31 \times 10^{-16}$ & 0.67 & 2.09 \\
II & $4.40 \times 10^{12}$ & 0.024 & $1.29 \times 10^{-13}$ & 0.14 & $5.58 \times 10^{-2}$ \\
III & $5.85 \times 10^{6}$ & 0.036 & 0.11 & 0.018 & $7.08 \times 10^{-8}$ \\
IV & $1.56 \times 10^{5}$ & 0.004 & 152 & $10^{-83}$ & $1.51 \times 10^{-9}$ \\
\hline \hline
\end{tabular}

TABLE II. The peak values of the power spectrum, the mass and abundance of PBHs, and the frequency of SIGWs.

where $\mu_{c}=9 \delta_{c} / 2 \sqrt{2}$. The fractional energy density of PBHs with the mass $M$ to DM is $[28,33]$

$$
\begin{aligned}
Y_{\mathrm{PBH}}(M)= & \frac{\beta(M)}{3.94 \times 10^{-9}}\left(\frac{\gamma}{0.2}\right)^{1 / 2}\left(\frac{g_{*}}{3.36}\right)^{-1 / 4} \\
& \times\left(\frac{0.12}{\Omega_{D M} h^{2}}\right)\left(\frac{M}{M_{\odot}}\right)^{-1 / 2},
\end{aligned}
$$

where $M_{\odot}=1.99 \times 10^{30} \mathrm{~kg}$ is the solar mass, $\gamma=0.2[141], \Omega_{\mathrm{DM}} h^{2}=0.12$ [40]. The effective degrees of freedom $g_{*}$ at the time of $\mathrm{PBH}$ formation is $g_{*}=106.75$ in the radiation dominated era. The mass of PBHs is

$$
\frac{M(k)}{M_{\odot}}=3.68\left(\frac{\gamma}{0.2}\right)\left(\frac{g_{*}}{3.36}\right)^{-1 / 6}\left(\frac{k}{10^{6} \mathrm{Mpc}^{-1}}\right)^{-2}
$$

Using the power spectrum obtained in Fig. 4, we calculate the mass and abundance of $\mathrm{PBHs}$ and display the results in Fig. 4 and Table II. A wide mass range of $\mathrm{PBH}$ is realized in our model, and we show four benchmark points where the PBH masses are around $\mathcal{O}\left(10^{-16} M_{\odot}\right), \mathcal{O}\left(10^{-12} M_{\odot}\right), \mathcal{O}\left(10^{-2} M_{\odot}\right)$ and $\mathcal{O}\left(10^{2} M_{\odot}\right)$. The PBHs with masses around $\mathcal{O}\left(10^{-16} M_{\odot}\right)$ and $\mathcal{O}\left(10^{-12} M_{\odot}\right)$ can make up almost all DM and the peak abundances are $Y_{\mathrm{PBH}} \simeq 1$. The $\mathrm{PBH}$ with the mass around $\mathcal{O}\left(10^{-2} M_{\odot}\right)$ only explains part of dark matter with $Y_{\mathrm{PBH}} \simeq 10^{-2}$. However, from Table II, we see that the PBH with the mass around $\mathcal{O}\left(10^{2} M_{\odot}\right)$ is hard to explain DM because of the significantly small value of $Y_{\mathrm{PBH}}$.

\section{Scalar induced gravitational wave}

Since the scalar perturbations and tensor perturbations are coupled at the second order, the large primordial curvature perturbation at small scales will induce second-order tensor 
perturbations. On CMB scale, the amplitude of tensor perturbation is much smaller than that of the scalar perturbation. The tensor-to-scalar ratio is constrained as $r_{0.05}<0.036$ (95\% CL) [115]. Therefore, the second-order tensor perturbations induced by the enhanced scalar perturbations may be larger than primordial GWs. The perturbed metric in the Newtonian gauge is given by

$$
\mathrm{d} s^{2}=a^{2}\left[-(1+2 \Phi) \mathrm{d} \tau^{2}+\left((1-2 \Psi) \delta_{i j}+\frac{1}{2} h_{i j}\right) \mathrm{d} x^{i} \mathrm{~d} x^{j}\right]
$$

In the following calculation, we will neglect the anisotropic stress, so $\Phi=\Psi$. The equation of motion for the tensor mode, $h_{k}(\tau)$, sourced by the scalar perturbation $\Phi_{k}(\tau)$, is

$$
h_{\mathbf{k}}^{\prime \prime}+2 \mathcal{H} h_{\mathbf{k}}^{\prime}+k^{2} h_{\mathbf{k}}=4 \mathcal{S}_{\mathbf{k}}
$$

where the source term is given by

$$
\begin{aligned}
\mathcal{S}_{k}= & \int \frac{\mathrm{d}^{3} \tilde{k}}{(2 \pi)^{3 / 2}} e_{i j}(k) \tilde{k}^{i} \tilde{k}^{j}\left[2 \Phi_{\tilde{k}} \Phi_{k-\tilde{k}}\right. \\
& \left.+\frac{4}{3(1+w) \mathcal{H}^{2}}\left(\Phi_{\tilde{k}}^{\prime}+\mathcal{H} \Phi_{\tilde{k}}\right)\left(\Phi_{k-\tilde{k}}^{\prime}+\mathcal{H} \Phi_{k-\tilde{k}}\right)\right],
\end{aligned}
$$

$w=p / \rho, e_{i j}(k)$ is the polarization tensor. In the radiation domination, the Bardeen potential $\Phi_{\mathbf{k}}(\tau)=\Phi(k \tau) \phi_{k}$ and the transfer function is

$$
\Phi(x)=\frac{9}{x^{2}}\left(\frac{\sin (x / \sqrt{3})}{x / \sqrt{3}}-\cos (x / \sqrt{3})\right),
$$

where $x=k \tau, 1 / \sqrt{3}$ is the sound speed of the radiation background. The fluctuation $\phi_{k}$ is related to the primordial curvature perturbation as

$$
\left\langle\phi_{\boldsymbol{k}} \phi_{\tilde{\boldsymbol{k}}}\right\rangle=\delta^{3}(\boldsymbol{k}+\tilde{\boldsymbol{k}}) \frac{2 \pi^{2}}{k^{3}}\left(\frac{3+3 w}{5+3 w}\right)^{2} \mathcal{P}_{\zeta} .
$$

The power spectrum of the tensor perturbation is defined as

$$
\left\langle h_{\boldsymbol{k}}(\tau) h_{\tilde{\boldsymbol{k}}}(\tau)\right\rangle=\delta^{3}(\boldsymbol{k}+\tilde{\boldsymbol{k}}) \frac{2 \pi^{2}}{k^{3}} \mathcal{P}_{h}(k, \tau) .
$$

After solving Eq. (41) with Green's function method, the power spectrum can be written as [71, 72, 84]

$$
\begin{aligned}
\mathcal{P}_{h}(k, \tau)= & 4 \int_{0}^{\infty} \mathrm{d} v \int_{|1-v|}^{1+v} \mathrm{~d} u \\
& \times\left(\frac{4 v^{2}-\left(1+v^{2}-u^{2}\right)^{2}}{4 u v}\right)^{2} \\
& \times I_{R D}^{2}(u, v, x) \mathcal{P}_{\zeta}(k v) \mathcal{P}_{\zeta}(k u),
\end{aligned}
$$


and the fractional energy density of the induced GWs is

$$
\Omega_{\mathrm{GW}}(\tau, k)=\frac{\rho_{G W}(\tau, k)}{\rho_{\text {tot }}(\tau)}=\frac{1}{24} \frac{k^{2}}{\mathcal{H}^{2}} \overline{\mathcal{P}_{h}(\tau, k)},
$$

where the overline denotes the oscillation average, $u=|\boldsymbol{k}-\tilde{\boldsymbol{k}}| / k$ and $v=\tilde{k} / k$. During the radiation era, the kernel function is [37, 71, 72, 84, 85]

$$
\overline{I_{R D}(u, v, x \rightarrow \infty)}=\frac{1}{2}\left(\frac{3\left(u^{2}+v^{2}-3\right)^{2}}{4 u^{3} v^{2} x}\right)^{2}\left(I_{s}+I_{c}\right),
$$

where $I_{s}=\pi^{2} \Theta(u+v-\sqrt{3})$ and $I_{c}=\left(\log \left|\frac{3-(u+v)^{2}}{3-(u-v)^{2}}\right|-\frac{4 u v}{u^{2}+v^{2}-3}\right)^{2}$.

Converting the wave number to frequency with $f_{\mathrm{GW}}=9.71586 \times 10^{-15} \mathrm{kHz} / \mathrm{Mpc}^{-1}$, the energy density of SIWGs against its frequency is plotted in Fig. 5. From Fig. 5, we see that the energy density of SIGWs illustrates some universal dependencies on the frequency [55, 142, 143]. The peak frequencies of these SIWGs are shown in Table II. The SIWGs generated in the models III and IV have a wide peak. This broad band with frequencies $f_{\mathrm{GW}} \sim\left[10^{-10}, 10^{2}\right] \mathrm{Hz}$, comes from the broad enhanced power spectrum with the wavenumber $k \sim\left[10^{4}, 10^{20}\right] \mathrm{Mpc}^{-1}$. The produced $\mathrm{PBH}$ with the stellar mass can constitute part of DM in our universe. The SIWGs in the nanohertz band can be interpreted as the stochastic GW background observed by the recent 12.5-yr PTA data released by the NANOGrav [22, 93]. The broad band SIGWs can be tested by multiband GW observations. The SIWGs generated in the model II have a peak at the frequency $f_{\mathrm{GW}} \sim \mathcal{O}\left(10^{-2}\right) \mathrm{Hz}$, which will be tested by the space-based GW detector, such as LISA, Taiji and TianQin. The SIWGs generated in the model I have a peak around the frequency $f_{\mathrm{GW}} \sim 10 \mathrm{~Hz}$ which will be tested by the future ground-based GW observatory.

\section{CONCLUSION}

We have investigated the formation of PBHs and SIGWs from the general no-scale supergravity inflationary model, inspired by the string model building. An inflection point is added by introducing an exponential term into Kähler potential. Thus, the amplitude of the primordial power spectrum is enhanced. To achieve the enhancement by the inflection point, the model parameters need to be fine-tuned by five decimal digits at most. The enhanced power spectra of primordial curvature perturbations can have both sharp and broad peaks. A wide mass range of $\mathrm{PBH}$ is realized in our model, and the frequencies of SIGWs 


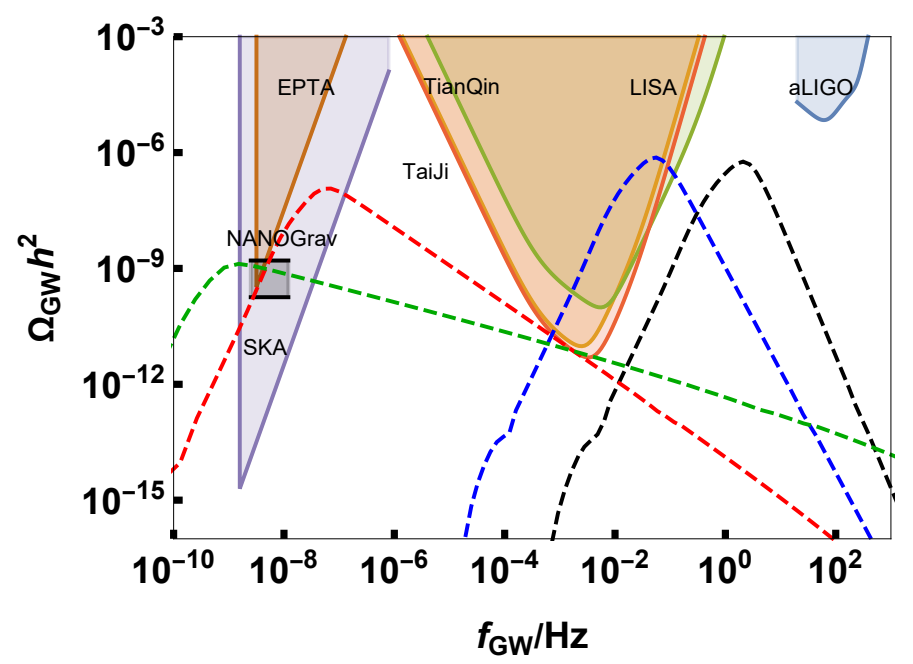

FIG. 5. The energy densities of SIGWs. The black, blue, red, and green dashed lines correspond to the models I, II, III, and IV, respectively. The shaded regions indicate sensitivity of GWs observatories, such as Square Kilometer Array (SKA) [144], European PTA (EPTA) [145-148], North American Nanohertz Observatory for GWs (NANOGrav) [92], Taiji [96], TianQin [97], Laser Interferometer Space Antenna (LISA) [95], and Advanced Laser Interferometer Gravitational Wave Observatory (aLIGO) [149, 150].

are ranged form nanohertz to kilohertz. We have shown four benchmark points where the PBH masses are around $\mathcal{O}\left(10^{-16} M_{\odot}\right), \mathcal{O}\left(10^{-12} M_{\odot}\right)$ and $\mathcal{O}\left(10^{-2} M_{\odot}\right)$ and $\mathcal{O}\left(10^{2} M_{\odot}\right)$. The SIGWs accompanied with the formation of stellar mass PBH can interpret the stochastic GW background detected by NANOGrav. The PBHs with masses around $\mathcal{O}\left(10^{-16} M_{\odot}\right)$ and $\mathcal{O}\left(10^{-12} M_{\odot}\right)$ can make up almost all DM and the accompanied SIGWs will be testable by the upcoming space-based GW observatories. The observations of both PBHs and SIGWs can test our model and inflationary physics. The broad band SIGWs generated in this model can be tested by multiband GW observations.

\section{ACKNOWLEDGMENTS}

This work is supported in part by the National Natural Science Foundation of China under Grants No. 11875062, No. 11875136, and No. 11947302, the Major Program of the National Natural Science Foundation of China under Grant No. 11690021, the Key Research Program of Frontier Science, CAS. This work was also supported in part by the Natural 
Science Basic Research Plan in Shanxi Province of China under Grant No. 2020JQ-804, and by the Shanxi Provincial Education Department under Grant No. 20JK0685.

[1] B. P. Abbott et al. (LIGO Scientific and Virgo Collaborations), Observation of gravitational waves from a binary black hole merger, Phys. Rev. Lett. 116, 061102 (2016).

[2] B. P. Abbott et al. (LIGO Scientific and Virgo Collaborations), GW151226: observation of gravitational waves from a 22-solar-mass binary black hole coalescence, Phys. Rev. Lett. 116, 241103 (2016).

[3] B. P. Abbott et al. (LIGO Scientific and Virgo Collaborations), GW170104: observation of a 50-solar-mass binary black hole coalescence at redshift 0.2, Phys. Rev. Lett. 118, 221101 (2017); 121, 129901(E) (2018).

[4] B. P. Abbott et al. (LIGO Scientific and Virgo Collaborations), GW170814: a three-detector observation of gravitational waves from a binary black bole coalescence, Phys. Rev. Lett. $119,141101(2017)$.

[5] B. P. Abbott et al. (LIGO Scientific and Virgo Collaborations), GW170817: observation of gravitational waves from a binary neutron star inspiral, Phys. Rev. Lett. 119, 161101 (2017).

[6] B. P. Abbott et al. (LIGO Scientific and Virgo Collaborations), GW170608: observation of a 19-solar-mass binary black hole coalescence, Astrophys. J. Lett. 851, L35 (2017).

[7] B. P. Abbott et al. (LIGO Scientific and Virgo Collaborations), GWTC-1: a gravitationalwave transient catalog of compact binary mergers observed by LIGO and Virgo during the first and second observing runs, Phys. Rev. X 9, 031040 (2019).

[8] B. P. Abbott et al. (LIGO Scientific and Virgo Collaborations), GW190425: observation of a compact binary coalescence with total mass $\sim 3.4 M_{\odot}$, Astrophys. J. Lett. 892, L3 (2020).

[9] R. Abbott et al. (LIGO Scientific and Virgo Collaborations), GW190412: observation of a binary-black-hole coalescence with asymmetric masses, Phys. Rev. D 102, 043015 (2020).

[10] R. Abbott et al. (LIGO Scientific and Virgo Collaborations), GW190814: gravitational waves from the coalescence of a 23 solar mass black hole with a 2.6 solar mass compact object, Astrophys. J. Lett. 896, L44 (2020).

[11] R. Abbott et al. (LIGO Scientific and Virgo Collaborations), GW190521: a binary black hole merger with a total mass of $150 M_{\odot}$, Phys. Rev. Lett. 125, 101102 (2020). 
[12] R. Abbott et al. (LIGO Scientific and Virgo Collaborations), GWTC-2: compact binary coalescences observed by LIGO and Virgo during the first half of the third observing run, Phys. Rev. X 11, 021053 (2021).

[13] R. Abbott et al. (LIGO Scientific, VIRGO and KAGRA Collaborations), GWTC-3: compact binary coalescences observed by LIGO and Virgo during the second part of the third observing run, arXiv:2111.03606.

[14] J. Garcia-Bellido, A. D. Linde, and D. Wands, Density perturbations and black hole formation in hybrid inflation, Phys. Rev. D 54, 6040 (1996).

[15] S. M. Leach and A. R. Liddle, Inflationary perturbations near horizon crossing, Phys. Rev. D 63, 043508 (2001).

[16] S. Bird, I. Cholis, J. B. Muñoz, Y. Ali-Haïmoud, M. Kamionkowski, E. D. Kovetz, A. Raccanelli, and A. G. Riess, Did LIGO detect dark matter?, Phys. Rev. Lett. 116, 201301 (2016).

[17] M. Sasaki, T. Suyama, T. Tanaka, and S. Yokoyama, Primordial black hole scenario for the gravitational-wave event GW150914, Phys. Rev. Lett. 117, 061101 (2016); 121, 059901(E) (2018) .

[18] V. De Luca, V. Desjacques, G. Franciolini, P. Pani, and A. Riotto, GW190521 mass gap event and the primordial black hole scenario, Phys. Rev. Lett. 126, 051101 (2021).

[19] J. Scholtz and J. Unwin, What if Planet 9 is a primordial black hole?, Phys. Rev. Lett. 125, 051103 (2020).

[20] V. Takhistov, G. M. Fuller, and A. Kusenko, Test for the origin of solar mass black holes, Phys. Rev. Lett. 126, 071101 (2021).

[21] V. De Luca, G. Franciolini, and A. Riotto, NANOGrav data hints at primordial black holes as dark matter, Phys. Rev. Lett. 126, 041303 (2021).

[22] V. Vaskonen and H. Veermäe, Did NANOGrav see a signal from primordial black hole formation?, Phys. Rev. Lett. 126, 051303 (2021).

[23] P. Ivanov, P. Naselsky, and I. Novikov, Inflation and primordial black holes as dark matter, Phys. Rev. D 50, 7173 (1994).

[24] P. H. Frampton, M. Kawasaki, F. Takahashi, and T. T. Yanagida, Primordial black holes as all dark matter, J. Cosmol. Astropart. Phys. 04 (2010) 023.

[25] K. M. Belotsky, A. D. Dmitriev, E. A. Esipova, V. A. Gani, A. V. Grobov, M. Y. Khlopov, A. A. Kirillov, S. G. Rubin, and I. V. Svadkovsky, Signatures of primordial black hole dark 
matter, Mod. Phys. Lett. A 29, 1440005 (2014).

[26] M. Y. Khlopov, S. G. Rubin, and A. S. Sakharov, Primordial structure of massive black hole clusters, Astropart. Phys. 23, 265 (2005).

[27] S. Clesse and J. García-Bellido, Massive primordial black holes from hybrid inflation as dark matter and the seeds of galaxies, Phys. Rev. D 92, 023524 (2015).

[28] B. Carr, F. Kuhnel, and M. Sandstad, Primordial black holes as dark matter, Phys. Rev. D 94, 083504 (2016).

[29] K. Inomata, M. Kawasaki, K. Mukaida, Y. Tada, and T. T. Yanagida, Inflationary primordial black holes as all dark matter, Phys. Rev. D 96, 043504 (2017).

[30] J. García-Bellido, Massive primordial black holes as dark matter and their detection with gravitational waves, J. Phys. Conf. Ser. 840, 012032 (2017).

[31] E. D. Kovetz, Probing primordial-black-hole dark matter with gravitational waves, Phys. Rev. Lett. 119, 131301 (2017).

[32] B. Carr and F. Kuhnel, Primordial black holes as dark matter: recent developments, Annu. Rev. Nucl. Part. Sci. 70, 355 (2020).

[33] B. Carr, K. Kohri, Y. Sendouda, and J. Yokoyama, Constraints on primordial black holes, arXiv:2002.12778.

[34] A. M. Green and B. J. Kavanagh, Primordial black holes as a dark matter candidate, J. Phys. G 48, 043001 (2021).

[35] S. Hawking, Gravitationally collapsed objects of very low mass, Mon. Not. R. Astron. Soc. 152, 75 (1971).

[36] B. J. Carr and S. W. Hawking, Black holes in the early Universe, Mon. Not. R. Astron. Soc. 168, 399 (1974).

[37] Y. Lu, Y. Gong, Z. Yi, and F. Zhang, Constraints on primordial curvature perturbations from primordial black hole dark matter and secondary gravitational waves, J. Cosmol. Astropart. Phys. 12, 031 (2019) .

[38] G. Sato-Polito, E. D. Kovetz, and M. Kamionkowski, Constraints on the primordial curvature power spectrum from primordial black holes, Phys. Rev. D 100, 063521 (2019).

[39] Y. Akrami et al. (Planck Collaboration), Planck 2018 results. X. Constraints on inflation, Astron. Astrophys. 641, A10 (2020). 
[40] N. Aghanim et al. (Planck Collaboration), Planck 2018 results. VI. Cosmological parameters, Astron. Astrophys. 641, A6 (2020); 652, C4(E) (2021).

[41] M. Kawasaki, A. Kusenko, Y. Tada, and T. T. Yanagida, Primordial black holes as dark matter in supergravity inflation models, Phys. Rev. D 94, 083523 (2016).

[42] H. Di and Y. Gong, Primordial black holes and second order gravitational waves from ultraslow-roll inflation, J. Cosmol. Astropart. Phys. 07 (2018) 007.

[43] J. Garcia-Bellido, M. Peloso, and C. Unal, Gravitational wave signatures of inflationary models from primordial black hole dark matter, J. Cosmol. Astropart. Phys. 09 (2017) 013.

[44] J. Garcia-Bellido and E. Ruiz Morales, Primordial black holes from single field models of inflation, Phys. Dark Univ. 18, 47 (2017).

[45] C. Germani and T. Prokopec, On primordial black holes from an inflection point, Phys. Dark Univ. 18, 6 (2017).

[46] J. M. Ezquiaga, J. Garcia-Bellido, and E. Ruiz Morales, Primordial black hole production in critical Higgs inflation, Phys. Lett. B 776, 345 (2018).

[47] F. Bezrukov, M. Pauly, and J. Rubio, On the robustness of the primordial power spectrum in renormalized Higgs inflation, J. Cosmol. Astropart. Phys. 02 (2018) 040.

[48] J. R. Espinosa, D. Racco, and A. Riotto, Cosmological signature of the standard model Higgs vacuum instability: primordial black holes as dark matter, Phys. Rev. Lett. 120, 121301 (2018).

[49] M. Cicoli, V. A. Diaz, and F. G. Pedro, Primordial black holes from string inflation, J. Cosmol. Astropart. Phys. 06 (2018) 034.

[50] T.-J. Gao and Z.-K. Guo, Primordial black hole production in inflationary models of supergravity with a single chiral superfield, Phys. Rev. D 98, 063526 (2018).

[51] G. Ballesteros, J. Beltran Jimenez, and M. Pieroni, Black hole formation from a general quadratic action for inflationary primordial fluctuations, J. Cosmol. Astropart. Phys. 06 (2019) 016.

[52] I. Dalianis, A. Kehagias, and G. Tringas, Primordial black holes from $\alpha$-attractors, J. Cosmol. Astropart. Phys. 01 (2019) 037.

[53] S. Passaglia, W. Hu, and H. Motohashi, Primordial black holes as dark matter through Higgs field criticality, Phys. Rev. D 101, 123523 (2020). 
[54] N. Bhaumik and R. K. Jain, Primordial black holes dark matter from inflection point models of inflation and the effects of reheating, J. Cosmol. Astropart. Phys. 01 (2020) 037.

[55] W.-T. Xu, J. Liu, T.-J. Gao, and Z.-K. Guo, Gravitational waves from double-inflection-point inflation, Phys. Rev. D 101, 023505 (2020).

[56] M. Braglia, D. K. Hazra, F. Finelli, G. F. Smoot, L. Sriramkumar, and A. A. Starobinsky, Generating PBHs and small-scale GWs in two-field models of inflation, J. Cosmol. Astropart. Phys. 08 (2020) 001.

[57] J. Lin, Q. Gao, Y. Gong, Y. Lu, C. Zhang, and F. Zhang, Primordial black holes and secondary gravitational waves from $k$ and $G$ inflation, Phys. Rev. D 101, 103515 (2020).

[58] Z. Yi, Y. Gong, B. Wang, and Z.-H. Zhu, Primordial black holes and secondary gravitational waves from the Higgs field, Phys. Rev. D 103, 063535 (2021).

[59] Z. Yi, Q. Gao, Y. Gong, and Z.-H. Zhu, Primordial black holes and scalar-induced secondary gravitational waves from inflationary models with a noncanonical kinetic term, Phys. Rev. D 103, 063534 (2021).

[60] Q. Gao, Y. Gong, and Z. Yi, Primordial black holes and secondary gravitational waves from natural inflation, Nucl. Phys. B 969, 115480 (2021).

[61] Q. Gao, Primordial black holes and secondary gravitational waves from chaotic inflation, Sci. China Phys. Mech. Astron. 64, 280411 (2021).

[62] C. Fu, P. Wu, and H. Yu, Primordial black holes from inflation with nonminimal derivative coupling, Phys. Rev. D 100, 063532 (2019).

[63] C. Fu, P. Wu, and H. Yu, Scalar induced gravitational waves in inflation with gravitationally enhanced friction, Phys. Rev. D 101, 023529 (2020).

[64] I. Dalianis, S. Karydas, and E. Papantonopoulos, Generalized non-minimal derivative coupling: application to inflation and primordial black hole production, J. Cosmol. Astropart. Phys. 06 (2020) 040.

[65] G. Ballesteros, J. Rey, M. Taoso, and A. Urbano, Primordial black holes as dark matter and gravitational waves from single-field polynomial inflation, J. Cosmol. Astropart. Phys. 07 (2020) 025.

[66] C.-Y. Chen, Threshold of primordial black hole formation in Eddington-inspired-Born-Infeld gravity, Int. J. Mod. Phys. D 30, 02 (2021). 
[67] E. McDonough, A. H. Guth, and D. I. Kaiser, Nonminimal couplings and the forgotten field of axion inflation, arXiv:2010.04179.

[68] G. Ballesteros and M. Taoso, Primordial black hole dark matter from single field inflation, Phys. Rev. D 97, 023501 (2018).

[69] S. Matarrese, S. Mollerach, and M. Bruni, Second order perturbations of the Einstein-de Sitter universe, Phys. Rev. D 58, 043504 (1998).

[70] S. Mollerach, D. Harari, and S. Matarrese, CMB polarization from secondary vector and tensor modes, Phys. Rev. D 69, 063002 (2004).

[71] K. N. Ananda, C. Clarkson, and D. Wands, The Cosmological gravitational wave background from primordial density perturbations, Phys. Rev. D 75, 123518 (2007).

[72] D. Baumann, P. J. Steinhardt, K. Takahashi, and K. Ichiki, Gravitational wave spectrum induced by primordial scalar perturbations, Phys. Rev. D 76, 084019 (2007).

[73] R. Saito and J. Yokoyama, Gravitational wave background as a probe of the primordial black hole abundance, Phys. Rev. Lett. 102, 161101 (2009); 107, 069901(E) (2011).

[74] R. Saito and J. Yokoyama, Gravitational-wave constraints on the abundance of primordial black holes, Prog. Theor. Phys. 123, 867 (2010); 126, 351(E) (2011).

[75] E. Bugaev and P. Klimai, Induced gravitational wave background and primordial black holes, Phys. Rev. D 81, 023517 (2010).

[76] E. Bugaev and P. Klimai, Constraints on the induced gravitational wave background from primordial black holes, Phys. Rev. D 83, 083521 (2011).

[77] L. Alabidi, K. Kohri, M. Sasaki, and Y. Sendouda, Observable spectra of induced gravitational waves from inflation, J. Cosmol. Astropart. Phys. 09 (2012) 017.

[78] N. Orlofsky, A. Pierce, and J. D. Wells, Inflationary theory and pulsar timing investigations of primordial black holes and gravitational waves, Phys. Rev. D 95, 063518 (2017).

[79] K. Inomata, M. Kawasaki, K. Mukaida, Y. Tada, and T. T. Yanagida, Inflationary primordial black holes for the LIGO gravitational wave events and pulsar timing array experiments, Phys. Rev. D 95, 123510 (2017).

[80] S.-L. Cheng, W. Lee, and K.-W. Ng, Primordial black holes and associated gravitational waves in axion monodromy inflation, J. Cosmol. Astropart. Phys. 07 (2018) 001.

[81] R.-G. Cai, S. Pi, and M. Sasaki, Gravitational waves induced by non-gaussian scalar perturbations, Phys. Rev. Lett. 122, 201101 (2019). 
[82] N. Bartolo, V. De Luca, G. Franciolini, M. Peloso, D. Racco, and A. Riotto, Testing primordial black holes as dark matter with LISA, Phys. Rev. D 99, 103521 (2019).

[83] N. Bartolo, V. De Luca, G. Franciolini, A. Lewis, M. Peloso, and A. Riotto, Primordial black hole dark matter: LISA serendipity, Phys. Rev. Lett. 122, 211301 (2019).

[84] K. Kohri and T. Terada, Semianalytic calculation of gravitational wave spectrum nonlinearly induced from primordial curvature perturbations, Phys. Rev. D 97, 123532 (2018).

[85] J. R. Espinosa, D. Racco, and A. Riotto, A cosmological signature of the SM Higgs instability: gravitational waves, J. Cosmol. Astropart. Phys. 09 (2018) 012.

[86] R.-G. Cai, S. Pi, S.-J. Wang, and X.-Y. Yang, Resonant multiple peaks in the induced gravitational waves, J. Cosmol. Astropart. Phys. 05 (2019) 013.

[87] R.-G. Cai, Z.-K. Guo, J. Liu, L. Liu, and X.-Y. Yang, Primordial black holes and gravitational waves from parametric amplification of curvature perturbations, J. Cosmol. Astropart. Phys. 06 (2020) 013.

[88] R.-G. Cai, S. Pi, S.-J. Wang, and X.-Y. Yang, Pulsar timing array constraints on the induced gravitational waves, J. Cosmol. Astropart. Phys. 10 (2019) 059.

[89] G. Domènech, Induced gravitational waves in a general cosmological background, Int. J. Mod. Phys. D 29, 2050028 (2020).

[90] R.-G. Cai, Y.-C. Ding, X.-Y. Yang, and Y.-F. Zhou, Constraints on a mixed model of dark matter particles and primordial black holes from the galactic $511 \mathrm{keV}$ line, J. Cosmol. Astropart. Phys. 03 (2021) 057.

[91] G. Domènech, S. Pi, and M. Sasaki, Induced gravitational waves as a probe of thermal history of the universe, J. Cosmol. Astropart. Phys. 08 (2020) 017.

[92] Z. Arzoumanian et al. (NANOGrav Collaboration), The NANOGrav 12.5 yr Data Set: Search for an isotropic stochastic gravitational-wave background, Astrophys. J. Lett. 905, L34 (2020).

[93] K. Kohri and T. Terada, Solar-mass primordial black holes explain NANOGrav hint of gravitational waves, Phys. Lett. B 813, 136040 (2021).

[94] T. Nakama, J. Silk, and M. Kamionkowski, Stochastic gravitational waves associated with the formation of primordial black holes, Phys. Rev. D 95, 043511 (2017).

[95] K. Danzmann, LISA: an ESA cornerstone mission for a gravitational wave observatory, Class. Quantum Grav. 14, 1399 (1997). 
[96] W.-R. Hu and Y.-L. Wu, The Taiji program in space for gravitational wave physics and the nature of gravity, Natl. Sci. Rev. 4, 685 (2017).

[97] J. Luo et al. (TianQin Collaboration), TianQin: a space-borne gravitational wave detector, Class. Quantum Grav. 33, 035010 (2016).

[98] J. Ellis, D. V. Nanopoulos, and K. A. Olive, Starobinsky-like inflationary models as avatars of no-scale supergravity, J. Cosmol. Astropart. Phys. 10(2013) 009.

[99] J. Ellis, D. V. Nanopoulos, and K. A. Olive, No-scale supergravity realization of the starobinsky model of inflation, Phys. Rev. Lett. 111, 111301 (2013); 111, 129902(E) (2013).

[100] D. Croon, J. Ellis, and N. E. Mavromatos, Wess-Zumino inflation in light of Planck, Phys. Lett. B 724, 165 (2013).

[101] S. Pi, Y.-l. Zhang, Q.-G. Huang, and M. Sasaki, Scalaron from $R^{2}$-gravity as a heavy field, J. Cosmol. Astropart. Phys. 05 (2018) 042.

[102] D. V. Nanopoulos, V. C. Spanos, and I. D. Stamou, Primordial black holes from no-scale supergravity, Phys. Rev. D 102, 083536 (2020).

[103] J. Ellis, D. V. Nanopoulos, K. A. Olive, and S. Verner, Unified no-scale attractors, J. Cosmol. Astropart. Phys. 09 (2019) 040.

[104] J. Ellis, M. A. G. Garcia, N. Nagata, N. D. V., K. A. Olive, and S. Verner, Building models of inflation in no-scale supergravity, Int. J. Mod. Phys. D 29, 2030011 (2020).

[105] I. D. Stamou, Mechanisms of producing primordial black holes by breaking the $S U(2,1) / S U(2) \times U(1)$ symmetry, Phys. Rev. D 103, 083512 (2021).

[106] E. Cremmer, S. Ferrara, C. Kounnas, and D. V. Nanopoulos, Naturally vanishing cosmological constant in $\mathrm{N}=1$ supergravity, Phys. Lett. B 133, 61 (1983).

[107] A. B. Lahanas and D. V. Nanopoulos, The road to no scale supergravity, Phys. Rep. 145, 1 (1987).

[108] E. Witten, Symmetry breaking patterns in superstring models, Nucl. Phys. B 258, 75 (1985).

[109] T.-J. Li, J. L. Lopez, and D. V. Nanopoulos, Compactifications of M theory and their phenomenological consequences, Phys. Rev. D 56, 2602 (1997).

[110] T.-J. Li, Compactification and supersymmetry breaking in M theory, Phys. Rev. D 57, 7539 (1998).

[111] H. Ooguri and C. Vafa, On the geometry of the string landscape and the swampland, Nucl. Phys. B 766, 21 (2007). 
[112] G. Obied, H. Ooguri, L. Spodyneiko, and C. Vafa, De Sitter space and the swampland, arXiv:1806.08362 [hep-th].

[113] M. K. Gaillard, H. Murayama, and K. A. Olive, Preserving flat directions during inflation, Phys. Lett. B 355, 71 (1995).

[114] G. A. Diamandis, J. R. Ellis, A. B. Lahanas, and D. V. Nanopoulos, Vanishing scalar masses in no scale supergravity, Phys. Lett. B 173, 303 (1986).

[115] P. A. R. Ade et al. (BICEP and Keck Array Collaborations), Improved constraints on primordial gravitational waves using Planck, WMAP, and BICEP/Keck observations through the 2018 observing season, Phys. Rev. Lett. 127, 151301 (2021).

[116] K. Inomata, E. McDonough, and W. Hu, Amplification of primordial perturbations from the rise or fall of the inflaton, arXiv:2110.14641.

[117] M. Sasaki, Large scale quantum fluctuations in the inflationary Universe, Prog. Theor. Phys. 76, 1036 (1986).

[118] V. F. Mukhanov, Quantum theory of gauge invariant cosmological perturbations, Sov. Phys. JETP 67, 1297 (1988).

[119] D. J. Fixsen, E. S. Cheng, J. M. Gales, J. C. Mather, R. A. Shafer, and E. L. Wright, The cosmic microwave background spectrum from the full COBE FIRAS data set, Astrophys. J. 473, $576(1996)$.

[120] B. J. Carr, K. Kohri, Y. Sendouda, and J. Yokoyama, New cosmological constraints on primordial black holes, Phys. Rev. D 81, 104019 (2010).

[121] P. W. Graham, S. Rajendran, and J. Varela, Dark matter triggers of supernovae, Phys. Rev. D 92, 063007 (2015).

[122] H. Niikura et al., Microlensing constraints on primordial black holes with Subaru/HSC Andromeda observations, Nat. Astron. 3, 524 (2019).

[123] K. Griest, A. M. Cieplak, and M. J. Lehner, New limits on primordial black hole dark matter from an analysis of Kepler source microlensing data, Phys. Rev. Lett. 111, 181302 (2013).

[124] P. Tisserand et al. (EROS-2 Collaboration), Limits on the macho content of the galactic halo from the EROS-2 survey of the magellanic clouds, Astron. Astrophys. 469, 387 (2007).

[125] M. Raidal, V. Vaskonen, and H. Veermäe, Gravitational waves from primordial black hole mergers, J. Cosmol. Astropart. Phys. 09 (2017) 037. 
[126] Y. Ali-Haïmoud, E. D. Kovetz, and M. Kamionkowski, Merger rate of primordial black-hole binaries, Phys. Rev. D 96, 123523 (2017).

[127] Y. Ali-Haïmoud and M. Kamionkowski, Cosmic microwave background limits on accreting primordial black holes, Phys. Rev. D 95, 043534 (2017).

[128] V. Poulin, P. D. Serpico, F. Calore, S. Clesse, and K. Kohri, CMB bounds on disk-accreting massive primordial black holes, Phys. Rev. D 96, 083524 (2017).

[129] P. Montero-Camacho, X. Fang, G. Vasquez, M. Silva, and C. M. Hirata, Revisiting constraints on asteroid-mass primordial black holes as dark matter candidates, J. Cosmol. Astropart. Phys. 08 (2019) 031.

[130] M. Shibata and M. Sasaki, Black hole formation in the Friedmann universe: formulation and computation in numerical relativity, Phys. Rev. D 60, 084002 (1999).

[131] A. G. Polnarev and I. Musco, Curvature profiles as initial conditions for primordial black hole formation, Class. Quantum Grav. 24, 1405 (2007).

[132] I. Musco, J. C. Miller, and A. G. Polnarev, Primordial black hole formation in the radiative era: investigation of the critical nature of the collapse, Class. Quantum Grav. 26, 235001 (2009).

[133] I. Musco and J. C. Miller, Primordial black hole formation in the early universe: critical behaviour and self-similarity, Class. Quantum Grav. 30, 145009 (2013).

[134] T. Harada, C.-M. Yoo, and K. Kohri, Threshold of primordial black hole formation, Phys. Rev. D 88, 084051 (2013); 89, 029903(E) (2014).

[135] C. Germani and I. Musco, Abundance of primordial black holes depends on the shape of the inflationary power spectrum, Phys. Rev. Lett. 122, 141302 (2019).

[136] Y. Tada and S. Yokoyama, Primordial black hole tower: dark matter, earth-mass, and LIGO black holes, Phys. Rev. D 100, 023537 (2019).

[137] A. Escrivà, C. Germani, and R. K. Sheth, Universal threshold for primordial black hole formation, Phys. Rev. D 101, 044022 (2020).

[138] C.-M. Yoo, T. Harada, and H. Okawa, Threshold of primordial black hole formation in nonspherical collapse, Phys. Rev. D 102, 043526 (2020).

[139] M. Solbi and K. Karami, Primordial black holes and induced gravitational waves in $k$ inflation, J. Cosmol. Astropart. Phys. 08 (2021) 056. 
[140] W. H. Press and P. Schechter, Formation of galaxies and clusters of galaxies by selfsimilar gravitational condensation, Astrophys. J. 187, 425 (1974).

[141] B. J. Carr, The primordial black hole mass spectrum, Astrophys. J. 201, 1 (1975).

[142] R.-G. Cai, S. Pi, and M. Sasaki, Universal infrared scaling of gravitational wave background spectra, Phys. Rev. D 102, 083528 (2020).

[143] C. Yuan, Z.-C. Chen, and Q.-G. Huang, Log-dependent slope of scalar induced gravitational waves in the infrared regions, Phys. Rev. D 101, 043019 (2020).

[144] C. J. Moore, R. H. Cole, and C. P. L. Berry, Gravitational-wave sensitivity curves, Class. Quant. Grav. 32, 015014 (2015).

[145] R. D. Ferdman et al., The european pulsar timing array: current efforts and a LEAP toward the future, Class. Quantum Grav. 27, 084014 (2010).

[146] G. Hobbs et al., The international pulsar timing array project: using pulsars as a gravitational wave detector, Class. Quantum Grav. 27, 084013 (2010).

[147] G. Hobbs, The parkes pulsar timing array, Class. Quantum Grav. 30, 224007 (2013).

[148] M. A. McLaughlin, The north american nanohertz observatory for gravitational waves, Class. Quantum Grav. 30, 224008 (2013).

[149] G. M. Harry (LIGO Scientific Collaboration), Advanced LIGO: the next generation of gravitational wave detectors, Class. Quantum Grav. 27, 084006 (2010).

[150] J. Aasi et al. (LIGO Scientific Collaboration), Advanced LIGO, Class. Quantum Grav. 32, 074001 (2015). 